\title{
Quantum Based Networks: Analysis of Quantum Teleportation Protocol and Entanglement Swapping
}

\author{
Preeti Kandwal ${ }^{1[0000-0002-4592-3699]}$, \\ William Joseph Spring $2[0000-0002-2251-2838]$ and \\ Hannan Xiao $3[0000-0003-2273-6679]$ \\ University of Hertfordshire, Hatfield, United Kingdom \\ p.kandwal@herts.ac. uk ${ }^{1}$ \\ j.spring@herts.ac. uk ${ }^{2}$ \\ h.xiao@herts.ac. uk ${ }^{3}$
}

\begin{abstract}
In this paper we consider the quantum teleportation and entanglement swapping protocols used in quantum based networks for passing information between a sender and receiver. For the teleportation protocol we observe and identify relationships that exist between Einstein-Podolsky-Rosen (EPR) Bell states employed as quantum resources, measured sender values and the gates employed at the receiver side. For the entanglement swapping protocol we consider input and output EPR states and the relationship between the two. We include a review of the concepts and our findings from the analysis carried out.
\end{abstract}

Keywords: Teleporation · Entanglement - Entanglement swapping · Bell states · Quantum networks and communication - Quantum applications

\section{Introduction}

Quantum networks have been in existence since DARPA's 2003 Quantum Network [19] and have experienced substantial development during the last two decades. Examples include SECOQC - Secure Communication based on Quantum Cryptography Network 33, the TOKYO Network 38 and the Generic Network and Networks in Classical communication [7]. Distances of $2000 \mathrm{~km}$ are now being achieved [2146], using entanglement, teleportation, and entanglement swapping. Recent developments in technology are opening up new and exciting possibilities for the practical application of quantum concepts to networks and distributed systems with the potential for realising a quantum based internet together with quantum based cloud resources [13|15|21|24|34].

Quantum teleportation is an important tool that is used in establishing a global platform for secure quantum networks and communication and distributed 
quantum applications [10|44. The sharing of information between a sender and a receiver via quantum based networks and distributed systems employs the use of quantum entanglement [1823, local operations with classical communication (LOCC) 8131/44 together with a classical communication channel. Teleportation is a fundamental resource which can be utilised in many applications such as quantum repeaters [12, quantum networks [7] and quantum computing based on measurements 36 .

From an experimental point of view we do not have pure quantum system due to decoherence; however, in theory quantum computing is considered a major breakthrough in technology. Quantum entanglement and quantum teleportation play a significant role in a variety of models for quantum communication and distributed networks 9113144]. Entanglement swapping [30|32] using polarised qubits [32] have laid the foundation for the development of quantum repeaters [12] enabling long distance quantum communication.

However, to the best of our knowledge, the analysis of the quantum teleportation and entanglement swapping protocols has not been generalised in the literature, although analysis of specific inputs have been presented. This motivates us to generalise the analysis for both protocols; such generalised analysis will facilitate further analysis of applications such as quantum-based networks with quantum repeaters that rely on the quantum teleportation and entanglement swapping protocols.

The rest of the paper is organised as below. In section 2 we briefly introduce the quantum teleportation protocol and present our generalised analysis and observations. In section 3, we present our generalised analysis of entanglement swapping and observations. Finally we conclude the paper in section 4 .

\section{Quantum Teleportation}

Quantum based research, both theoretical and experimental have now moved into a 'second global wave' with academia and industry combining to develop and advance quantum technology. Companies such as IBM [1, Google [2], Toshiba [3, Intel [4] and Microsoft [5] are each actively involved in the quantum revolution. With time and technology, one of the most outstanding achievement has been in the distance achieved for networks using teleportation [29/35/42 45]46]. In 1998, the first successful teleportation was observed approximately across a distance of one meter 42 at the California Institute of Technology. In 2006, Quantum teleportation between different quantum systems (light and matter) was experimentally achieved by Sherson et al, [39, increasing the possibility for improved quantum memories 26]40. Such developments involving memory and distance are now possible together with the possibility for the development of a quantum internet [27. Success followed in 2012 when an experiment 29] achieved over 143 kilometers of quantum teleportation between the two Canary 
Islands of La Palma and Tenerife. This led to the establishment of metropolitan area networks using optic modes leading to unconditional security [20] over long distance quantum networks.

Recent breakthroughs [45] in the area of long distance quantum teleportation have improved quantum communication achieving success in establishing quantum communication across a free space distance of 1200 kilometers (by free space is meant no interference from the environment which is practically difficult to achieve, and is carried in space). Using free space for the purpose of quantum communication reduces the chances of channel loss because the travel path for each photon is predominantly in empty space [37, as a result no disturbance is experienced in establishing quantum communication.

\subsection{Teleportation}

We commence this section with the brief introduction of the teleportation protocol itself. The quantum teleportation protocol is used in quantum networks for teleporting an unknown state from one location to another location. It is used to teleport or transfer any unknown state between a pair of users sharing an entangled Bell state.

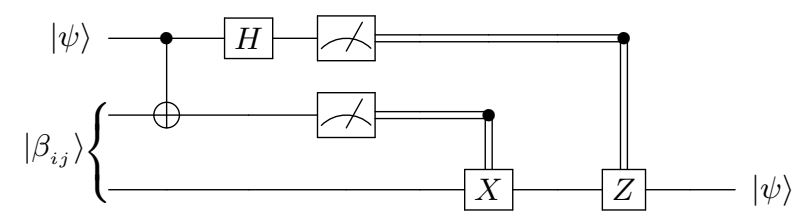

Fig. 1. Quantum Teleportation Protocol 17.

Generating Bell states: Bell states are the maximally entangled states of two qubits. Bell state is generated using a Hadamard gate, $\mathbb{H}$ along with a Controlled NOT or CNOT gate. The following circuit diagram illustrates how one can generate Bell states.

For any two qubits $|i\rangle$ and $|j\rangle$ with $i, j \in 0,1$, we define the associated Bell state as:

$$
\left|\beta_{i j}\right\rangle=\frac{1}{\sqrt{2}}\left(|0 j\rangle+(-1)^{i}|1 \bar{j}\rangle\right)
$$

in which $\bar{j}$ represents the opposite of $j$, e.g., if $j=0$ then $\bar{j}=1$ and vice versa. We therefore have four Bell states in total in which $i j \in\{00,01,10,11\}$. 


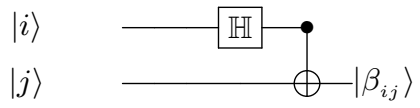

Fig. 2. Bell states generation [17]

For example, if we input the qubits $|0\rangle$ and $|0\rangle$ to the circuit, then the Hadamard gate changes the state of $|00\rangle$ to $\frac{1}{\sqrt{2}}((|0\rangle+|1\rangle)|0\rangle)=\frac{1}{\sqrt{2}}(|00\rangle+|10\rangle)$, to which the CNOT gate is applied generating $\frac{1}{\sqrt{2}}(|00\rangle+|11\rangle)$.

The four Bell states are

$$
\begin{aligned}
& \left|\beta_{00}\right\rangle=\frac{1}{\sqrt{2}}(|00\rangle+|11\rangle) \\
& \left|\beta_{01}\right\rangle=\frac{1}{\sqrt{2}}(|01\rangle+|10\rangle) \\
& \left|\beta_{10}\right\rangle=\frac{1}{\sqrt{2}}(|00\rangle-|11\rangle) \\
& \left|\beta_{11}\right\rangle=\frac{1}{\sqrt{2}}(|01\rangle-|10\rangle)
\end{aligned}
$$

\subsection{A generalised analysis of the teleportation protocol}

In this section we present our analysis and findings as we generalise the teleportation protocol with each of the four Bell state inputs and compare the patterns that emerge in the output.

Theorem 1. Let $A$ denotes a sender and $B$ denotes a receiver. Let $|\psi\rangle=$ $\alpha|0\rangle+\beta|1\rangle$ denotes an unknown qubit state that $A$ wants to send to $B$. Let $\left|\beta_{i j}\right\rangle$ with $i, j \in\{0,1\}$ denote a general Bell state. Then following measurement at $A$ with respect to the basis $B=\{|k l\rangle\}_{k, l} \in\{0,1\}$ the photon at $B$ is found to be in one of four states

$$
|\bar{\psi}\rangle= \begin{cases}\alpha|j\rangle \pm(-1)^{i} \beta|\bar{j}\rangle, & i, j \in\{0,2\} \\ \beta|j\rangle \pm(-1)^{i} \alpha|\bar{j}\rangle, & i, j \in\{1,3\}\end{cases}
$$

resulting in recovery of $|\psi\rangle$ at the receiver's side following the application of $\mathbb{Z}^{i+k} \mathbb{X}^{j+l}$ to the receiver's part of the shared Bell state resource.

Proof. Using equation (1) and applying the operator $(\mathbb{H} \otimes \mathbb{I})_{0} C N O T$ at the senders side to the unknown state $|\psi\rangle$ with shared Bell state resource $\left|\beta_{i j}\right\rangle$ generates the given result for $|\bar{\psi}\rangle$ in quantum teleportation protocol.

$\left|\psi_{1}\right\rangle=|\psi\rangle \otimes\left|\beta_{i j}\right\rangle$

$\left|\psi_{1}\right\rangle=(\alpha|0\rangle+\beta|1\rangle) \cdot \frac{1}{\sqrt{2}}\left(|0 j\rangle+(-1)^{i}|1 \bar{j}\rangle\right)$ 


$$
\begin{aligned}
& =\frac{1}{\sqrt{2}}\left\{\alpha|0\rangle\left(|0 j\rangle+(-1)^{i}|1 \bar{j}\rangle\right)+\beta|1\rangle\left(|0 j\rangle+(-1)^{i}|1 \bar{j}\rangle\right)\right\} \\
& =\frac{1}{\sqrt{2}}\left(\alpha|0\rangle|0 j\rangle+(-1)^{i} \alpha|0\rangle|1 \bar{j}\rangle+\beta|1\rangle|0 j\rangle+(-1)^{i} \beta|1\rangle|1 \bar{j}\rangle\right) \\
& \left|\psi_{2}\right\rangle=(\mathbb{C N O T} \otimes \mathbb{I})\left|\psi_{1}\right\rangle \\
& =\frac{1}{\sqrt{2}}\left(\alpha|0\rangle\left(|0 j\rangle+(-1)^{i}|1 \bar{j}\rangle\right)+\beta|1\rangle\left(|1 j\rangle+(-1)^{i}|0 \bar{j}\rangle\right)\right) \\
& \left|\psi_{3}\right\rangle=(\mathbb{H} \otimes \mathbb{I} \otimes \mathbb{I})\left|\psi_{2}\right\rangle \\
& =\frac{1}{\sqrt{2}} \cdot \frac{1}{\sqrt{2}}\left(\alpha(|0\rangle+|1\rangle)\left(|0 j\rangle+(-1)^{i}|1 \bar{j}\rangle\right)+\beta(|0\rangle-|1\rangle)\left(|1 j\rangle+(-1)^{i}|0 \bar{j}\rangle\right)\right) \\
& \frac{1}{2}\left(\alpha|00 j\rangle+(-1)^{i} \alpha|01 \bar{j}\rangle+\alpha 10 j+(-1)^{i} \alpha|11 \bar{j}\rangle+\beta|01 j\rangle+(-1)^{i} \beta|00 \bar{j}\rangle-\beta|11 j\rangle-\right. \\
& \left.(-1)^{i} \beta|10 \bar{j}\rangle\right) \\
& =\frac{1}{2}\left(|00\rangle\left(\alpha|j\rangle+(-1)^{i} \beta|\bar{j}\rangle\right)+|01\rangle\left((-1)^{i} \alpha|\bar{j}\rangle+\beta|j\rangle\right)+|10\rangle\left(\alpha|j\rangle-(-1)^{i} \beta|\bar{j}\rangle\right)+\right. \\
& \left.|11\rangle\left((-1)^{i} \alpha|\bar{j}\rangle-\beta|j\rangle\right)\right) \\
& =\frac{1}{2}\left(|00\rangle\left(\alpha|j\rangle+(-1)^{i} \beta|\bar{j}\rangle\right)+|01\rangle\left(\beta|j\rangle+(-1)^{i} \alpha|\bar{j}\rangle\right)+|10\rangle\left(\alpha|j\rangle-(-1)^{i} \beta|\bar{j}\rangle\right)-\right. \\
& \left.|11\rangle(\beta|j\rangle)-(-1)^{i} \alpha|\bar{j}\rangle\right)
\end{aligned}
$$

We now measure with respect to the basis $\mid\{k, l\rangle\}_{k, l \in\{0,1\}}$ identifying the corresponding receiver states as shown in Table 1

Table 1. Quantum Teleportation Protocol with classical values and corresponding outputs.

\begin{tabular}{|c|c|c|}
\hline Classical (k,l) & $|k l\rangle$ & Corresponding operation \\
\hline$(0,0)$ & $|00\rangle$ & $\left(\alpha|j\rangle+(-1)^{i} \beta|\bar{j}\rangle\right)$ \\
\hline$(0,1)$ & $|01\rangle$ & $\left(\beta|j\rangle+(-1)^{i} \alpha|\bar{j}\rangle\right)$ \\
\hline$(1,0)$ & $|10\rangle$ & $\left(\alpha|j\rangle-(-1)^{i} \beta|\bar{j}\rangle\right)$ \\
\hline$(1,1)$ & $|11\rangle$ & $-\left(\beta|j\rangle-(-1)^{i} \alpha|\bar{j}\rangle\right)$ \\
\hline
\end{tabular}

Based on the above generalisation we note that the following formula for teleportation holds.

$$
|\psi\rangle \stackrel{\left|\beta_{i j}\right\rangle}{\longmapsto}|k l\rangle \mathbb{X}^{j+l} \mathbb{Z}^{i+k}
$$

where $\left|\beta_{i j}\right\rangle$ is any of the Bell state; $|i, j\rangle$ represents the qubits in Bell state; and $k, l$ represents the classical values after measurement. Hence for any $\left|\beta_{i j}\right\rangle$ and $(k, l)$, Bob (receiver) needs to apply $\mathbb{X}^{j+l} \mathbb{Z}^{i+k}$; we note that the superscripts are 
mod2. By substituting the $i j$ values of the entangled Bell state in the Table 1 the reciever can reconstruct the original quantum state, $|\psi\rangle$.

Example 1. Let us consider the case, where $\left|\beta_{i j}\right\rangle=\left|\beta_{\text {oo }}\right\rangle$ and using table 1 we receive the below information on following the protocol:

$|00\rangle\left(\alpha|j\rangle+(-1)^{i} \beta|\bar{j}\rangle\right) \Longrightarrow|00\rangle\left(\alpha|0\rangle+(-1)^{0} \beta|1\rangle\right)=|00\rangle(\alpha|0\rangle+\beta|1\rangle)=$ $|00\rangle|\psi\rangle$

$|01\rangle\left(\beta|j\rangle+(-1)^{i} \alpha|\bar{j}\rangle\right) \Longrightarrow|01\rangle\left(\beta|0\rangle+(-1)^{0} \alpha|1\rangle\right)=|01\rangle(\beta|0\rangle+\alpha|1\rangle)=$ $|01\rangle \mathbb{X}|\psi\rangle$

$|10\rangle\left(\alpha|j\rangle-(-1)^{i} \beta|\bar{j}\rangle\right) \Longrightarrow|10\rangle\left(\alpha|0\rangle-(-1)^{0} \beta|1\rangle\right)=|10\rangle(\alpha|0\rangle-\beta|1\rangle)=$ $|10\rangle \mathbb{Z}|\psi\rangle$

$|11\rangle\left\{-\left(\beta|j\rangle+(-1)^{i} \alpha|\bar{j}\rangle\right)\right\} \Longrightarrow|11\rangle\left\{-\left(\beta|0\rangle-(-1)^{0} \alpha|1\rangle\right)\right\}=|11\rangle\{-(\beta|0\rangle-$ $\alpha|1\rangle)\}=|11\rangle(\alpha|1\rangle-\beta|0\rangle)=|11\rangle \mathbb{X} \mathbb{Z}|\psi\rangle$

\subsection{Observations}

Combining all four Bell states together we obtain the following table:

Table 2. Quantum Teleportation Protocol for all four Bell states with classical values and corresponding quantum operators.

\begin{tabular}{|c|c|c|c|c|c|c|c|c|}
\hline$\left|\beta_{i j}\right\rangle$ & \multicolumn{2}{|c|}{ Input Bell state $\left|\beta_{00}\right|$} & \multicolumn{2}{|c|}{ Input Bell state $\mid \beta_{01}$} & \multicolumn{2}{|c|}{$\mid$ Input Bell state $\left|\beta_{10}\right\rangle$} & \multicolumn{2}{|c|}{$\mid$ Input Bell state $\left|\beta_{11}\right\rangle$} \\
\hline$|i j\rangle$ & $|00\rangle$ & $|\psi\rangle$ & $|01\rangle$ & $|\psi\rangle$ & $|10\rangle$ & $|\psi\rangle$ & || $11\rangle$ & $|\psi\rangle$ \\
\hline$|i \bar{j}\rangle$ & $|01\rangle$ & $\mathbb{X}|\psi\rangle$ & $|00\rangle$ & $\mathbb{X}|\psi\rangle$ & $|11\rangle$ & $\mathbb{X}|\psi\rangle$ & $|10\rangle$ & $\mathbb{X}|\psi\rangle$ \\
\hline$|\bar{i} j\rangle$ & $|10\rangle$ & $\mathbb{Z}|\psi\rangle$ & $|11\rangle$ & $\mathbb{Z}|\psi\rangle$ & $|00\rangle$ & $\mathbb{Z}|\psi\rangle$ & $|01\rangle$ & $\mathbb{Z}|\psi\rangle$ \\
\hline$|\bar{i} \overline{i j}\rangle$ & || $11\rangle$ & $\mathbb{X} \mathbb{Z}|\psi\rangle$ & $|10\rangle$ & $\mathbb{\mathbb { Z } Z}|\psi\rangle$ & $|01\rangle$ & $\mathbb{X Z}|\psi\rangle$ & $|00\rangle$ & $\overline{\mathbb{Z} Z}|\psi\rangle$ \\
\hline
\end{tabular}

We observe the patterns from Table 2 for the teleportation protocol and find that given an entangled state $\left|\beta_{i j}\right\rangle$ and measurement outcomes $(\mathrm{k}, \mathrm{l})$ on the senders side, the corresponding state on the receivers side would be as follows:

Table 3. General form of teleportation.

\begin{tabular}{|l|c|c|c|c|}
\hline$\left|\beta_{i j}\right\rangle$ & $k=0, l=0$ & $k=0, l=1$ & $k=1, l=0$ & $k=1, l=1$ \\
\hline$\left|\beta_{00}\right\rangle$ & $|\psi\rangle$ & $\mathbb{X}|\psi\rangle$ & $\mathbb{Z}|\psi\rangle$ & $\mathbb{X} \mathbb{Z}|\psi\rangle$ \\
\hline$\left|\beta_{01}\right\rangle$ & $\mathbb{X}|\psi\rangle$ & $|\psi\rangle$ & $\mathbb{X} Z|\psi\rangle$ & $\mathbb{Z}|\psi\rangle$ \\
\hline$\left|\beta_{10}\right\rangle$ & $\mathbb{Z}|\psi\rangle$ & $\mathbb{X} \mathbb{Z}|\psi\rangle$ & $|\psi\rangle$ & $\mathbb{X}|\psi\rangle$ \\
\hline$\left|\beta_{11}\right\rangle$ & $\mathbb{X} \mathbb{Z}|\psi\rangle$ & $\mathbb{Z}|\psi\rangle$ & $\mathbb{X}|\psi\rangle$ & $|\psi\rangle$ \\
\hline
\end{tabular}


Note: Based on the qubits used in our Bell state and measured values $(k, l)$, we can define the corresponding quantum operator at the receiver's end.

We can apply the required quantum operator and recreate the information based on the shared Bell states and corresponding measurement outcomes.

Table 4. Operators applied to recreate the information in specific cases of Bell states.

\begin{tabular}{|l|l|l|l|l|l|c|}
\hline$\left|\beta_{i j}\right\rangle$ & $\left|\beta_{00}\right\rangle$ & $\left|\beta_{01}\right\rangle$ & $\left|\beta_{10}\right\rangle$ & $\left|\beta_{11}\right\rangle$ & Teleported state \\
\hline$|i j\rangle$ & $|00\rangle$ & $|01\rangle$ & $|10\rangle$ & $|11\rangle$ & $\alpha|0\rangle+\beta|1\rangle \rightarrow|\psi\rangle$ \\
\hline$|i \bar{j}\rangle$ & $|01\rangle$ & $|00\rangle$ & $|11\rangle$ & $|10\rangle$ & $\alpha|1\rangle+\beta|0\rangle \rightarrow \mathbb{X}|\psi\rangle$ \\
\hline$|\bar{i} j\rangle$ & $|10\rangle$ & $|11\rangle$ & $|00\rangle$ & $|01\rangle$ & $\alpha|0\rangle-\beta|1\rangle \rightarrow \mathbb{Z}|\psi\rangle$ \\
\hline$|\bar{i} \bar{j}\rangle$ & $|11\rangle$ & $|10\rangle$ & $|01\rangle$ & $|00\rangle$ & $\alpha|1\rangle-\beta|0\rangle \rightarrow \mathbb{X} \mathbb{Z}|\psi\rangle$ \\
\hline
\end{tabular}

Or we can rewrite table 3 in the form of below relationship that holds for input and corresponding output:

$$
\left|\beta_{i j}\right\rangle \stackrel{\mathrm{QTP}}{\longrightarrow}|i j\rangle|\psi\rangle \pm|i \bar{j}\rangle \mathbb{X}|\psi\rangle \pm|\bar{i} j\rangle \mathbb{Z}|\psi\rangle \pm|\bar{i} \bar{j}\rangle \mathbb{X} \mathbb{Z}|\psi\rangle
$$

Where $\bar{i}$ and $\bar{j}$ are the negation of $i$ and $j$ respectively, i.e., if $i=0$ then $\bar{i}=1$ and vice versa and similarly for $j$, if $j=0$ then $\bar{j}=1$ and vice versa.

Example Let $i=j=0$ then

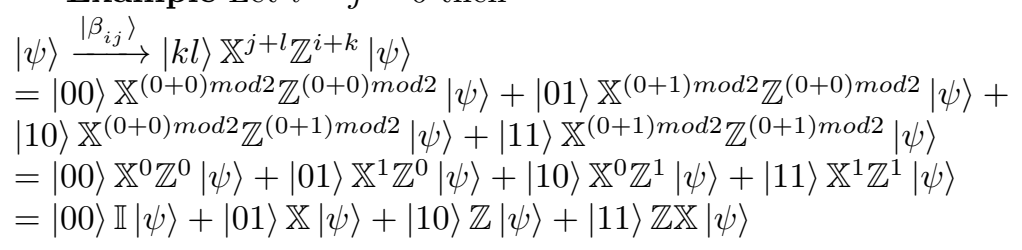

\section{$3 \quad$ Entanglement Swapping}

\subsection{Introduction}

We now present a brief introduction to the entanglement swapping protocol and swapping process itself. Entanglement swapping [614 25/28|47] refers to the activity of swapping entanglement from a pre-existing pair of entangled photons to a corresponding pair of non entangled photons. This is a key resource used in quantum networks to establish entanglement between two quantum entities such as a pair of routers on a quantum network.

Suppose we have 4 photons namley A, B, C and D with two entangled states. Photon A and photon B are entangled in one state and photons $\mathrm{C}$ and D are entangled in another state. We assume that there exists Bell state entanglement 
between the pairs A-B and C-D. This implies that any measurement on photon A will affect the state of photon $\mathrm{B}$ and vice-versa and similarly any observation on photon $\mathrm{C}$ will affect the state of photon $\mathrm{D}$ and vice versa.

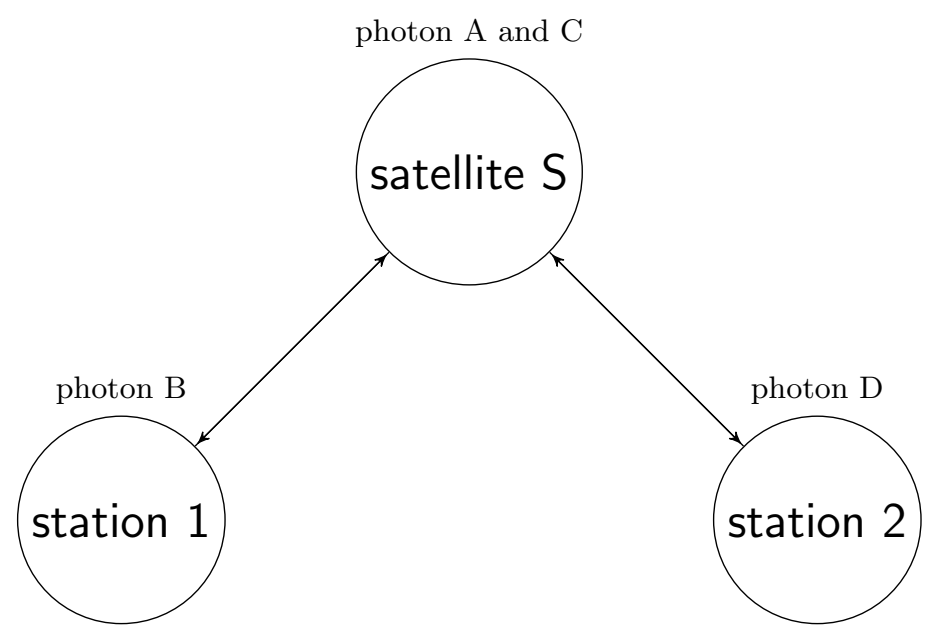

Fig. 3. Pre Entanglement Swapping: entanglement exists between photons A-B and C-D.

In entanglement swapping, by operating a Bell measurement on $\mathrm{A}$ and $\mathrm{C}$ we establish entanglement between B and D. We note that in establishing entanglement between $\mathrm{B}$ and $\mathrm{D}$ we lose the previously shared entanglement links between $\mathrm{A}$ and $\mathrm{B}$ and between $\mathrm{C}$ and $\mathrm{D}$.

\subsection{A generalised analysis of entanglement swapping}

Theorem 2. Let satellite $S$ and station 1 and station 2 be as shown in figure 3. Let $\left|\beta_{i j}\right\rangle_{A B}$ denote one pair of an arbitrary entangled Bell states between photons $A$ and $B$ and $\left|\beta_{k l}\right\rangle_{C D}$ denote a second pair of arbitray entangled Bell states between photons $C$ and $D$. Then the state for the system is given by:

$$
|\psi\rangle_{A B C D}=\left|\beta_{i j}\right\rangle_{A B} \cdot\left|\beta_{k l}\right\rangle_{C B}
$$



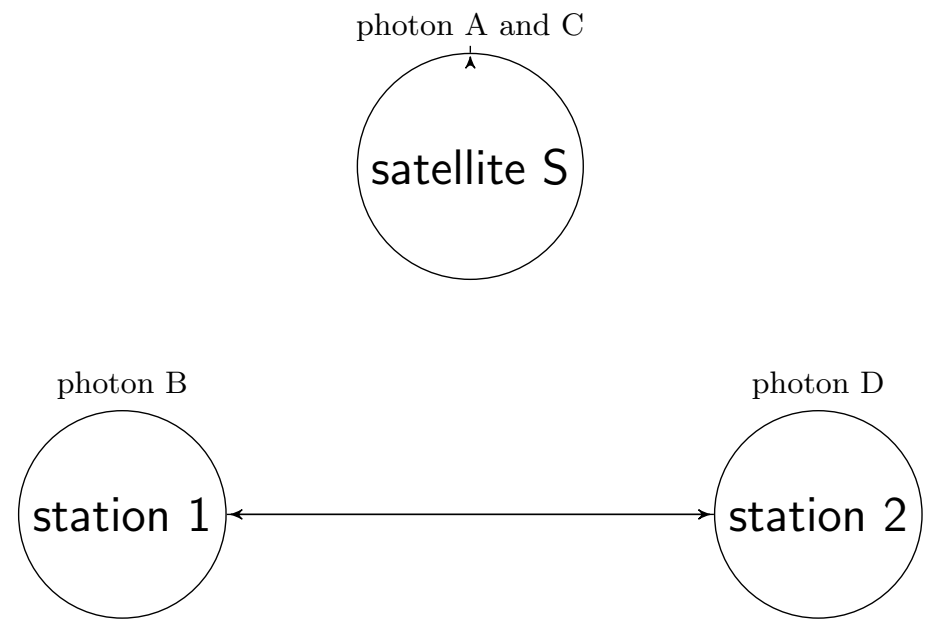

Fig. 4. Post Entanglement Swapping: initial entangled states are now separable and new entangled states established between photons A-C and B-D respectively.

$=\left\{\begin{array}{cc}\left|\beta_{00}\right\rangle_{A C}\left|\beta_{i+k, l}\right\rangle_{B D}+(-1)^{k}\left|\beta_{01}\right\rangle_{A C}\left|\beta_{i+k, \bar{l}}\right\rangle_{B D}+\left|\beta_{10}\right\rangle_{A C}\left|\beta_{i+k+1, l}\right\rangle_{B D}+ \\ (-1)^{k}\left|\beta_{11}\right\rangle_{A C}\left|\beta_{i+k+1, \bar{l}}\right\rangle_{B D} & (\text { for } j=0) \\ (-1)^{i+k}\left|\beta_{00}\right\rangle_{A C}\left|\beta_{i+k, \bar{l}}\right\rangle_{B D}+\left|\beta_{01}\right\rangle_{A C}\left|\beta_{i+k, \bar{l}}\right\rangle_{B D}+(-1)^{i+k+1}\left|\beta_{10}\right\rangle_{A C}\left|\beta_{i+k+1, \bar{l}}\right\rangle_{B D}+ \\ (-1)^{k}\left|\beta_{11}\right\rangle_{A C}\left|\beta_{i+k+1, l}\right\rangle_{B D} & \text { (for } j=1)\end{array}\right.$

Hence measuring with respect to the Bell basis at the satellite leads to a corresponding Bell state between the two stations in terms of photon $B$ and photon $D$.

Proof. Let $\left|\psi_{A B C D}\right\rangle$ denote the state for the four photons at A, B, C and D. Since there exists an initial entanglement between photons A-B and C-D which is defined by two Bell states, $\left|\beta_{i j}\right\rangle_{A B}$ and $\left|\beta_{k l}\right\rangle_{C D}$ respectively, it follows that $\left|\psi_{A B C D}\right\rangle=\left|\psi_{A B}\right\rangle \cdot\left|\psi_{C D}\right\rangle$

$=\frac{1}{\sqrt{2}}\left|\beta_{i j}\right\rangle_{A B} \cdot \frac{1}{\sqrt{2}}\left|\beta_{k l}\right\rangle_{C D}$

$=\frac{1}{2}\left(|0 j\rangle_{A B}+(-1)^{i}|1 \bar{j}\rangle_{A B}\right) \cdot\left(|0 l\rangle_{C D}+(-1)^{k}|1 \bar{l}\rangle_{C D}\right)$

$=\frac{1}{2}\left(|0 j\rangle_{A B}|0 l\rangle_{C D}+(-1)^{k}|0 j\rangle_{A B}|1 \bar{l}\rangle_{C D}+(-1)^{i}|1 \bar{j}\rangle_{A B}|0 l\rangle_{C D}+(-1)^{i+k}|1 \bar{j}\rangle_{A B}|1 \bar{l}\rangle_{C D}\right)$ 
NOTE: For simplicity we omit the constant scalar multiples but note that we will be working with normalised states throughout.

$=|0 j 0 l\rangle_{A B C D}+(-1)^{k}|0 j 1 \bar{l}\rangle_{A B C D}+(-1)^{i}|1 \bar{j} 0 l\rangle_{A B C D}+(-1)^{i+k}|1 \bar{j} 1 \bar{l}\rangle_{A B C D}$

$=\left(|00\rangle_{A C}|j l\rangle_{B D}+(-1)^{k}|01\rangle_{A C}|j \bar{l}\rangle_{B D}+(-1)^{i}|10\rangle_{A C}|\bar{j} l\rangle_{B D}+(-1)^{i+k}|11\rangle_{A C}|\bar{j} \bar{l}\rangle_{B D}\right)$

In terms of the states at the satellite (photons $\mathrm{A}$ and $\mathrm{C}$ ) we obtain the following:

$=\left(\left|\beta_{00}\right\rangle+\left|\beta_{10}\right\rangle\right)_{A C}|j l\rangle_{B D}+(-1)^{k}\left(\left|\beta_{01}\right\rangle+\left|\beta_{11}\right\rangle\right)_{A C}|j \bar{l}\rangle_{B D}+$

$(-1)^{i}\left(\left|\beta_{01}\right\rangle-\left|\beta_{11}\right\rangle\right)_{A C}|\bar{j} l\rangle_{B D}+(-1)^{i+k}\left(\left|\beta_{00}\right\rangle-\left|\beta_{10}\right\rangle\right)_{A C}|\bar{j} \bar{l}\rangle_{B D}$

$\left.=\left|\beta_{00}\right\rangle_{A C}|j l\rangle_{B D}+\left|\beta_{10}\right\rangle_{A C}|j l\rangle_{B D}+(-1)^{k}\left|\beta_{01}\right\rangle_{A C}|j \bar{l}\rangle_{B D}+(-1)^{k}\left|\beta_{11}\right\rangle\right)_{A C}|j \bar{l}\rangle_{B D}+$

$(-1)^{i}\left|\beta_{01}\right\rangle|\bar{j} l\rangle_{B D}-(-1)^{i}\left|\beta_{11}\right\rangle_{A C}|\bar{j} l\rangle_{B D}+(-1)^{i+k}\left|\beta_{00}\right\rangle_{A C}|\bar{j} \bar{l}\rangle_{B D}-$

$(-1)^{i+k}\left|\beta_{10}\right\rangle_{A C}|\bar{j} \bar{l}\rangle_{B D}$

$=\left(\left|\beta_{00}\right\rangle_{A C}|j l\rangle_{B D}+(-1)^{i+k}\left|\beta_{00}\right\rangle_{A C}|\bar{j} \bar{l}\rangle_{B D}\right)+\left((-1)^{k}\left|\beta_{01}\right\rangle_{A C}|j \bar{l}\rangle_{B D}+(-1)^{i}\right.$

$\left.\left|\beta_{01}\right\rangle_{A C}|\bar{j} l\rangle_{B D}\right)+\left(\left|\beta_{10}\right\rangle_{A C}|j l\rangle_{B D}-(-1)^{i+k}\left|\beta_{10}\right\rangle_{A C}|\bar{j} \bar{l}\rangle_{B D}\right)+\left((-1)^{k}\left|\beta_{11}\right\rangle_{A C}|j \bar{l}\rangle_{B D}-\right.$ $\left.(-1)^{i}\left|\beta_{11}\right\rangle_{A C}|\bar{j} l\rangle_{B D}\right)$

$=\left|\beta_{00}\right\rangle_{A C}\left(|j l\rangle_{B D}+(-1)^{i+k}|\bar{j} \bar{l}\rangle_{B D}\right)+\left|\beta_{01}\right\rangle_{A C}\left((-1)^{k}|j \bar{l}\rangle_{B D}+(-1)^{i}|\bar{j} l\rangle_{B D}\right)+$ $\left|\beta_{10}\right\rangle_{A C}\left(|j l\rangle_{B D}-(-1)^{i+k}|\bar{j} \bar{l}\rangle_{B D}\right)+\left|\beta_{11}\right\rangle_{A C}\left((-1)^{k}|j \bar{l}\rangle_{B D}-(-1)^{i}|\bar{j} l\rangle_{B D}\right)$

$=\left|\beta_{00}\right\rangle_{A C}\left(|j l\rangle+(-1)^{i+k}|\bar{j} \bar{l}\rangle\right)_{B D}+\left|\beta_{01}\right\rangle_{A C}\left((-1)^{k}|j \bar{l}\rangle+(-1)^{i}|\bar{j} l\rangle\right)_{B D}+\left|\beta_{10}\right\rangle_{A C}(|j l\rangle-$ $\left.(-1)^{i+k}|\bar{j} \bar{l}\rangle\right)_{B D}+\left|\beta_{11}\right\rangle_{A C}\left((-1)^{k}|j \bar{l}\rangle-(-1)^{i}|\bar{j} l\rangle\right)_{B D}$

$$
\begin{aligned}
& =\left|\beta_{00}\right\rangle_{A C}\left(|j l\rangle+(-1)^{i+k}|\bar{j} \bar{l}\rangle\right)_{B D}+\left|\beta_{01}\right\rangle_{A C}\left((-1)^{k}|j \bar{l}\rangle+(-1)^{i}|\bar{j} l\rangle\right)_{B D}+ \\
& \left|\beta_{10}\right\rangle_{A C}\left(|j l\rangle+(-1)^{i+k+1}|\bar{j} \bar{l}\rangle\right)_{B D}+\left|\beta_{11}\right\rangle_{A C}\left((-1)^{k}|j \bar{l}\rangle+(-1)^{i+1}|\bar{j} l\rangle\right)_{B D}
\end{aligned}
$$

From equation (7) we note the following two cases in terms of the Bell states where all subscripts (sum of subscripts) are mod 2.

1. For $j=0$

$$
\begin{aligned}
& |\psi\rangle_{A B C D}=\left|\beta_{00}\right\rangle\left(\left|\beta_{(i+k), l}\right\rangle\right)+\left|\beta_{01}\right\rangle\left((-1)^{k}\left|\beta_{(i+k), \bar{l}}\right\rangle\right)+\left|\beta_{10}\right\rangle\left(\left|\beta_{(i+k+1), l}\right\rangle\right)+ \\
& \left|\beta_{11}\right\rangle\left((-1)^{k}\left|\beta_{(i+k+1), \bar{l}}\right\rangle\right)
\end{aligned}
$$

2. For $j=1$

$$
\begin{aligned}
& |\psi\rangle_{A B C D}=\left|\beta_{00}\right\rangle\left((-1)^{i+k}\left|\beta_{(i+k), \bar{l}}\right\rangle\right)+\left|\beta_{01}\right\rangle\left(\left|\beta_{(i+k), l}\right\rangle\right)+\left|\beta_{10}\right\rangle\left((-1)^{i+k+1}\left|\beta_{(i+k+1), \bar{l}}\right\rangle\right)+ \\
& \left|\beta_{11}\right\rangle\left((-1)^{i+1}\left|\beta_{(i+k+1), l}\right\rangle\right)
\end{aligned}
$$

We present the possible input and output Bell states that occur in Table 5 
Table 5. All possible inputs for entanglement swapping using Bell states with swapped output.

\begin{tabular}{|c|c|c|c|c|}
\hline \multirow{2}{*}{\begin{tabular}{|c|} 
Initial Bell states \\
$\left|\beta_{00}\right\rangle_{A B}\left|\beta_{00}\right\rangle_{C D}$ \\
\end{tabular}} & \multicolumn{4}{|c|}{ Result (Swapped entanglement) } \\
\hline & $\left|\beta_{00}\right\rangle_{A C}\left|\beta_{00}\right\rangle_{B D}$ & $\left|\beta_{01}\right\rangle_{A C}\left|\beta_{01}\right\rangle_{B D}$ & $\left|\beta_{10}\right\rangle_{A C}\left|\beta_{10}\right\rangle_{B D}$ & $\left|\beta_{11}\right\rangle_{A C}\left|\beta_{11}\right\rangle_{B D}$ \\
\hline$\left|\beta_{00}\right\rangle_{A B}\left|\beta_{01}\right\rangle_{C D}$ & $\left|\beta_{00}\right\rangle_{A C}\left|\beta_{01}\right\rangle_{B D}$ & $\left|\beta_{01}\right\rangle_{A C}\left|\beta_{00}\right\rangle_{B D}$ & $\left|\beta_{10}\right\rangle_{A C}\left|\beta_{11}\right\rangle_{B D}$ & $\left|\beta_{11}\right\rangle_{A C}\left|\beta_{10}\right\rangle_{B D}$ \\
\hline$\left|\beta_{00}\right\rangle_{A B}\left|\beta_{10}\right\rangle_{C D}$ & $\left|\beta_{00}\right\rangle_{A C}\left|\beta_{10}\right\rangle_{B D}$ & $-\left|\beta_{01}\right\rangle_{A C}\left|\beta_{11}\right\rangle_{B D}$ & $\left|\beta_{10}\right\rangle_{A C}\left|\beta_{00}\right\rangle_{B D}$ & $-\left|\beta_{11}\right\rangle_{A C}\left|\beta_{01}\right\rangle_{B D}$ \\
\hline$\left|\beta_{00}\right\rangle_{A B}\left|\beta_{11}\right\rangle_{C D}$ & $\left|\beta_{00}\right\rangle_{A C}\left|\beta_{11}\right\rangle_{B D}$ & $-\left|\beta_{01}\right\rangle_{A C}\left|\beta_{10}\right\rangle_{B D}$ & $\left|\beta_{10}\right\rangle_{A C}\left|\beta_{01}\right\rangle_{B D}$ & $-\left|\beta_{11}\right\rangle_{A C}\left|\beta_{00}\right\rangle_{B D}$ \\
\hline$\left|\beta_{01}\right\rangle_{A B}\left|\beta_{00}\right\rangle_{C D}$ & $\left|\beta_{00}\right\rangle_{A C}\left|\beta_{01}\right\rangle_{B D}$ & $\left|\beta_{01}\right\rangle_{A C}\left|\beta_{00}\right\rangle_{B D}$ & $-\left|\beta_{10}\right\rangle_{A C}\left|\beta_{11}\right\rangle_{B D}$ & $-\left|\beta_{11}\right\rangle_{A C}\left|\beta_{10}\right\rangle_{B D}$ \\
\hline$\left|\beta_{01}\right\rangle_{A B}\left|\beta_{01}\right\rangle_{C D}$ & $\left|\beta_{00}\right\rangle_{A C}\left|\beta_{00}\right\rangle_{B D}$ & $\left|\beta_{01}\right\rangle_{A C}\left|\beta_{01}\right\rangle_{B D}$ & $-\left|\beta_{10}\right\rangle_{A C}\left|\beta_{10}\right\rangle_{B D}$ & $-\left|\beta_{11}\right\rangle_{A C}\left|\beta_{11}\right\rangle_{B D}$ \\
\hline$\left|\beta_{01}\right\rangle_{A B}\left|\beta_{10}\right\rangle_{C D}$ & $\left.|-| \beta_{00}\right\rangle_{A C}\left|\beta_{11}\right\rangle_{B D}$ & $\left|\beta_{01}\right\rangle_{A C}\left|\beta_{10}\right\rangle_{B D}$ & $\left|\beta_{10}\right\rangle_{A C}\left|\beta_{01}\right\rangle_{B D}$ & $-\left|\beta_{11}\right\rangle_{A C}\left|\beta_{00}\right\rangle_{B D}$ \\
\hline$\left|\beta_{01}\right\rangle_{A B}\left|\beta_{11}\right\rangle_{C D}$ & $\left.|-| \beta_{00}\right\rangle_{A C}\left|\beta_{10}\right\rangle_{B D}$ & $\left|\beta_{01}\right\rangle_{A C}\left|\beta_{11}\right\rangle_{B D}$ & $\left|\beta_{10}\right\rangle_{A C}\left|\beta_{00}\right\rangle_{B D}$ & $-\left|\beta_{11}\right\rangle_{A C}\left|\beta_{01}\right\rangle_{B D}$ \\
\hline$\left|\beta_{10}\right\rangle_{A B}\left|\beta_{00}\right\rangle_{C D}$ & $\left.|-| \beta_{00}\right\rangle_{A C}\left|\beta_{10}\right\rangle_{B D}$ & $-\left|\beta_{01}\right\rangle_{A C}\left|\beta_{11}\right\rangle_{B D}$ & $\left|\beta_{10}\right\rangle_{A C}\left|\beta_{00}\right\rangle_{B D}$ & $\left|\beta_{11}\right\rangle_{A C}\left|\beta_{01}\right\rangle_{B D}$ \\
\hline$\left|\beta_{10}\right\rangle_{A B}\left|\beta_{01}\right\rangle_{C D}$ & $\left|\beta_{00}\right\rangle_{A C}\left|\beta_{11}\right\rangle_{B D}$ & $\left|\beta_{01}\right\rangle_{A C}\left|\beta_{10}\right\rangle_{B D}$ & $\left|\beta_{10}\right\rangle_{A C}\left|\beta_{01}\right\rangle_{B D}$ & $\left|\beta_{11}\right\rangle_{A C}\left|\beta_{00}\right\rangle_{B D}$ \\
\hline$\left|\beta_{10}\right\rangle_{A B}\left|\beta_{10}\right\rangle_{C D}$ & $\left|\beta_{00}\right\rangle_{A C}\left|\beta_{00}\right\rangle_{B D}$ & $-\left|\beta_{01}\right\rangle_{A C}\left|\beta_{01}\right\rangle_{B D}$ & $\left|\beta_{10}\right\rangle_{A C}\left|\beta_{10}\right\rangle_{B D}$ & $-\left|\beta_{11}\right\rangle_{A C}\left|\beta_{11}\right\rangle_{B D}$ \\
\hline$\left|\beta_{10}\right\rangle_{A B}\left|\beta_{11}\right\rangle_{C D}$ & $\left|\beta_{00}\right\rangle_{A C}\left|\beta_{01}\right\rangle_{B D}$ & $-\left|\beta_{01}\right\rangle_{A C}\left|\beta_{00}\right\rangle_{B D}$ & $\left|\beta_{10}\right\rangle_{A C}\left|\beta_{11}\right\rangle_{B D}$ & $-\left|\beta_{11}\right\rangle_{A C}\left|\beta_{10}\right\rangle_{B D}$ \\
\hline$\left|\beta_{11}\right\rangle_{A B}\left|\beta_{00}\right\rangle_{C D}$ & $\left.|-| \beta_{00}\right\rangle_{A C}\left|\beta_{11}\right\rangle_{B D}$ & $-\left|\beta_{01}\right\rangle_{A C}\left|\beta_{10}\right\rangle_{B D}$ & $\left|\beta_{10}\right\rangle_{A C}\left|\beta_{01}\right\rangle_{B D}$ & $\left|\beta_{11}\right\rangle_{A C}\left|\beta_{00}\right\rangle_{B D}$ \\
\hline$\left|\beta_{11}\right\rangle_{A B}\left|\beta_{01}\right\rangle_{C D}$ & $\left.|-| \beta_{00}\right\rangle_{A C}\left|\beta_{10}\right\rangle_{B D}$ & $-\left|\beta_{01}\right\rangle_{A C}\left|\beta_{11}\right\rangle_{B D}$ & $\left|\beta_{10}\right\rangle_{A C}\left|\beta_{00}\right\rangle_{B D}$ & $\left|\beta_{11}\right\rangle_{A C}\left|\beta_{01}\right\rangle_{B D}$ \\
\hline$\left|\beta_{11}\right\rangle_{A B}\left|\beta_{10}\right\rangle_{C D}$ & $\left|\beta_{00}\right\rangle_{A C}\left|\beta_{01}\right\rangle_{B D}$ & $-\left|\beta_{01}\right\rangle_{A C}\left|\beta_{00}\right\rangle_{B D}$ & $-\left|\beta_{10}\right\rangle_{A C}\left|\beta_{11}\right\rangle_{B D}$ & $\left|\beta_{11}\right\rangle_{A C}\left|\beta_{10}\right\rangle_{B D}$ \\
\hline$\left|\beta_{11}\right\rangle_{A B}\left|\beta_{11}\right\rangle_{C D}$ & $\left|\beta_{00}\right\rangle_{A C} \mid \beta$ & $-\left|\beta_{01}\right\rangle_{A C} \mid \beta_{01}$ & $\left.|-| \beta_{10}\right\rangle_{A C}\left|\beta_{10}\right\rangle_{B}$ & $\left|\beta_{11}\right\rangle_{A C}\left|\beta_{11}\right\rangle_{B D}$ \\
\hline
\end{tabular}

We formulate the entanglement swapping as follows:

$$
|\psi\rangle_{A B . C D}=\sum_{k=0}^{3}\left|\beta_{(i+k) \bmod 4}\right\rangle_{A C} \cdot\left|\beta_{\left(j+(-1)^{(i+j) . k) \bmod 4}\right.}\right\rangle_{B D}
$$

We note $|\psi\rangle=\left|\beta_{i}\right\rangle \cdot\left|\beta_{j}\right\rangle=\left|\beta_{i}\right\rangle_{A B} \cdot\left|\beta_{j}\right\rangle_{C D}$ where $i$ and $j$ corresponds to pairs of Bell states for which we wish to use entanglement swapping. $i, j \in\{0,1,2,3\}$, in which $\{0,1,2,3\}$ are decimal representations corresponding to the binary values $\{00,01,10,11\}$ respectively. Additions for subscripts are calculated in mod4.

\subsection{Observations}

We note that we swapped the entanglement from particles A-B and C-D to particles $\mathrm{A}-\mathrm{C}$ and $\mathrm{B}-\mathrm{D}$ and lost the initial entanglement between $\mathrm{AB}$ and $\mathrm{CD}$. We observe from Table 5 that we have 16 combinations of inputs for which we can perform entanglement swapping, but irrespective of the different inputs we 
obtain only 4 possible unique outputs. The results are presented in Table 6 . We also note that we get the outcome as a combination of input state (i.e, the output is similar to the input entangled states). For four different inputs we have the same output, a combination of the four states; for example,

$\left|\beta_{00}\right\rangle \cdot\left|\beta_{00}\right\rangle,\left|\beta_{01}\right\rangle \cdot\left|\beta_{01}\right\rangle,\left|\beta_{10}\right\rangle \cdot\left|\beta_{10}\right\rangle,\left|\beta_{11}\right\rangle \cdot\left|\beta_{11}\right\rangle=$ $\left|\beta_{00}\right\rangle \cdot\left|\beta_{00}\right\rangle \pm\left|\beta_{01}\right\rangle \cdot\left|\beta_{01}\right\rangle \pm\left|\beta_{10}\right\rangle \cdot\left|\beta_{10}\right\rangle \pm\left|\beta_{11}\right\rangle \cdot\left|\beta_{11}\right\rangle$.

We now consider which swapped state is going to be utilised between B-D for the purpose of communication from the initial combination of Bell states, between $\mathrm{A}$ and $\mathrm{B}$ and between $\mathrm{C}$ and $\mathrm{D}$.

The following table presents pairs of input states and their corresponding possible output (swapped) states subject to measurement at AC.

Table 6. Combination of different entangled Bell states input and corresponding swapped entangled states.

\begin{tabular}{|c|c|c|c|c|}
\hline \multicolumn{4}{|c|}{ Initial Entangled states $\left(\left|\beta_{A B}\right\rangle \cdot\left|\beta_{C D}\right\rangle\right)$} & Swapped Entangled states $\left(\left|\beta_{A C}\right\rangle \cdot\left|\beta_{B D}\right\rangle\right)$ \\
\hline$\left|\beta_{00}\right\rangle \cdot\left|\beta_{00}\right\rangle \mid$ & $\left|\beta_{01}\right\rangle \cdot\left|\beta_{01}\right\rangle$ & $\left|\beta_{10}\right\rangle \cdot\left|\beta_{10}\right\rangle$ & $\left|\beta_{11}\right\rangle \cdot\left|\beta_{11}\right\rangle$ & $\left|\beta_{00}\right\rangle \cdot\left|\beta_{00}\right\rangle \pm\left|\beta_{01}\right\rangle \cdot\left|\beta_{01}\right\rangle \pm\left|\beta_{10}\right\rangle \cdot\left|\beta_{10}\right\rangle \pm\left|\beta_{11}\right\rangle \cdot\left|\beta_{11}\right\rangle$ \\
\hline$\left|\beta_{00}\right\rangle \cdot\left|\beta_{01}\right\rangle \mid$ & $\left|\beta_{01}\right\rangle \cdot\left|\beta_{00}\right\rangle$ & $\left|\beta_{10}\right\rangle \cdot\left|\beta_{11}\right\rangle$ & $\left|\beta_{11}\right\rangle \cdot\left|\beta_{10}\right\rangle$ & $\left|\beta_{00}\right\rangle \cdot\left|\beta_{01}\right\rangle \pm\left|\beta_{01}\right\rangle \cdot\left|\beta_{00}\right\rangle$ \\
\hline$\left|\beta_{00}\right\rangle \cdot\left|\beta_{10}\right\rangle \mid$ & $\left|\beta_{01}\right\rangle \cdot\left|\beta_{11}\right\rangle$ & $\left|\beta_{10}\right\rangle \cdot\left|\beta_{00}\right\rangle$ & $\left|\beta_{11}\right\rangle \cdot\left|\beta_{01}\right\rangle$ & $\left|\beta_{00}\right\rangle \cdot\left|\beta_{10}\right\rangle \pm\left|\beta_{01}\right\rangle \cdot\left|\beta_{11}\right\rangle \pm\left|\beta_{10}\right\rangle \cdot\left|\beta_{00}\right\rangle \pm\left|\beta_{11}\right\rangle \cdot\left|\beta_{01}\right\rangle$ \\
\hline$\left.\beta_{00}\right\rangle \cdot\left|\beta_{11}\right\rangle \mid$ & $\left|\beta_{01}\right\rangle \cdot\left|\beta_{10}\right\rangle \mid$ & $\left|\beta_{10}\right\rangle \cdot\left|\beta_{01}\right\rangle$ & $\left|\beta_{11}\right\rangle \cdot\left|\beta_{00}\right\rangle$ & $\left|\beta_{00}\right\rangle \cdot\left|\beta_{11}\right\rangle \pm\left|\beta_{01}\right\rangle \cdot\left|\beta_{10}\right\rangle \pm\left|\beta_{10}\right\rangle \cdot\left|\beta_{01}\right\rangle \pm\left|\beta_{11}\right\rangle \cdot\left|\beta_{00}\right\rangle$ \\
\hline
\end{tabular}

Replacing each Bell state with its corresponding subscript value, (that is, $\left|\beta_{00}\right\rangle$ becomes $0,\left|\beta_{01}\right\rangle$ becomes $1,\left|\beta_{10}\right\rangle$ becomes 2 and $\left|\beta_{11}\right\rangle$ becomes 3$)$, we observe:

Table 7. Updated table 6 using the terminology used in proposed formula.

\begin{tabular}{|c|c|c|c|c|}
\hline \multicolumn{4}{|c|}{ Initial Entangled states } & Swapped Entangled states \\
\hline 0.0 & 1.1 & 2.2 & 3.3 & $0.0+1.1+2.2+3.3$ \\
\hline 0.1 & 1.0 & 2.3 & 3.2 & $0.1+1.0+2.3+3.2$ \\
\hline 0.2 & 1.3 & 2.0 & 3.1 & $0.2+1.3+2.0+3.1$ \\
\hline 0.3 & 1.2 & 2.1 & 3.0 & $0.3+1.2+2.1+3.0$ \\
\hline
\end{tabular}

Note: We have dropped the 'minus' sign because it does not have any observable effect [31].

Throughout we note as AC increases from 0 to 3, BD increases in steps of 1 mod 4 for the first and third lines in Table 7 but decreases in steps of $1 \bmod 4$ for the other two lines. 
Example 2. For any given pair of initial entangled states we can deduce the swapped entangled states between the particles by utilising the formula given in equation (8).

For example

$$
\begin{aligned}
& |i\rangle_{A B} \cdot|j\rangle_{C D}=|0\rangle_{A B} \cdot|0\rangle_{C D} \\
& =\left|\beta_{00}\right\rangle_{A B} \cdot\left|\beta_{00}\right\rangle_{C D} \\
& =\left|\beta_{(0+0) \bmod 4}\right\rangle_{A C} \cdot\left|\beta_{(0+(-1)(0+0) .0) \bmod 4}\right\rangle_{B D}+\left|\beta_{(0+1) \bmod 4}\right\rangle_{A C} \cdot\left|\beta_{(0+(-1)(0+0) .1) \bmod 4}\right\rangle_{B D}
\end{aligned}
$$

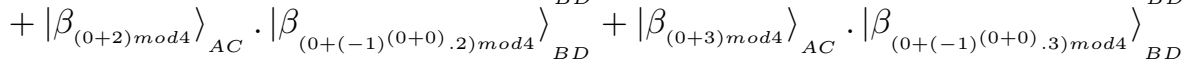

$$
\begin{aligned}
& =\left|\beta_{\text {omod4 }}\right\rangle_{A C} \cdot\left|\beta_{\text {Omod } 4}\right\rangle_{B D}+\left|\beta_{1 \bmod 4}\right\rangle_{A C}^{B D} \cdot\left|\beta_{1 \bmod 4}\right\rangle_{B D}+\left|\beta_{2 \bmod 4}\right\rangle_{A C} \cdot\left|\beta_{2 \bmod 4}\right\rangle_{B D}+ \\
& \left|\beta_{3 \bmod 4}\right\rangle_{A C} \cdot\left|\beta_{3 \bmod 4}\right\rangle_{B D} \\
& =\left|\beta_{0}\right\rangle_{A C}^{A C} \cdot\left|\beta_{0}\right\rangle_{B D}+\left|\beta_{1}\right\rangle_{A C} \cdot\left|\beta_{1}\right\rangle_{B D}+\left|\beta_{2}\right\rangle_{A C} \cdot\left|\beta_{2}\right\rangle_{B D}+\left|\beta_{3}\right\rangle_{A C} \cdot\left|\beta_{3}\right\rangle_{B D} \\
& =\left|\beta_{00}\right\rangle_{A C} \cdot\left|\beta_{00}\right\rangle_{B D}+\left|\beta_{01}\right\rangle_{A C} \cdot\left|\beta_{01}\right\rangle_{B D}+\left|\beta_{10}\right\rangle_{A C} \cdot\left|\beta_{10}\right\rangle_{B D}+\left|\beta_{11}\right\rangle_{A C} \cdot\left|\beta_{11}\right\rangle_{B D}
\end{aligned}
$$

Similary we can check all other pairs and get final entangled states as given in Table 5

\section{Conclusion, Future work and Applications}

In this work we have analysed the teleportation and entanglement swapping protocols and have identified relationships between inputs and their corresponding outputs. We have summarised our observations in both tables and as generalised formulae, relating inputs to outputs. We are currently developing applications that employ the above results within a quantum network setting. These includes ring signatures, voting protocols 16/22 41/43 and long distance communication. Details will follow shortly.

\section{References}

1. Meet ibm q. https://www.research.ibm.com/ibm-q/

2. Quantum a.i. research at google. https://research.google.com/pubs/ QuantumAI.html/

3. Quantum information. https://www.toshiba.eu/eu/ Cambridge-Research-Laboratory/Quantum-Information/

4. Qutech quantum institute enters into collaboration with intel. https://www.tudelft.nl/en/2015/tu-delft/ qutech-quantum-institute-enters-into-collaboration-with-intel

5. Station $\mathrm{q}$ - worldwide consortium for the advancement of topological quantum computation. https://stationq.microsoft.com/

6. Quantum teleportation of patterns of light. https://www.sciencedaily.com/ releases/2017/09/170921121147.htm (2017)

7. Quantum network - wikipedia. https://en.wikipedia.org/wiki/Quantum_ network (2018) 
8. Bennett, C.H., Brassard, G., Crépeau, C., Jozsa, R., Peres, A., Wootters, W.K.: Teleporting an unknown quantum state via dual classical and einstein-podolskyrosen channels. Physical review letters 70(13), 1895 (1993)

9. Bennett, C.H., Shor, P.W.: Quantum information theory. IEEE transactions on information theory 44(6), 2724-2742 (1998)

10. Bouwmeester, D., Pan, J.W., Mattle, K., Eibl, M., Weinfurter, H., Zeilinger, A.: Experimental quantum teleportation. Nature 390(6660), 575-579 (1997)

11. Braunstein, S.L., Van Loock, P.: Quantum information with continuous variables. Reviews of Modern Physics 77(2), 513 (2005)

12. Briegel, H.J., Dür, W., Cirac, J.I., Zoller, P.: Quantum repeaters: the role of imperfect local operations in quantum communication. Physical Review Letters 81(26), 5932 (1998)

13. Castelvecchi, D., et al.: Quantum cloud goes commercial (2017)

14. De Riedmatten, H., Marcikic, I., Van Houwelingen, J., Tittel, W., Zbinden, H., Gisin, N.: Long-distance entanglement swapping with photons from separated sources. Physical Review A 71(5), 050302 (2005)

15. Devitt, S.J.: Performing quantum computing experiments in the cloud. Physical Review A 94(3), 032329 (2016)

16. Dolev, S., Pitowsky, I., Tamir, B.: A quantum secret ballot. arXiv preprint quantph/0602087 (2006)

17. Eastin, B., Flammia, S.T.: Q-circuit tutorial. arXiv preprint quant-ph/0406003 (2004)

18. Eisert, J., Plenio, M.: Introduction to the basics of entanglement theory in continuous-variable systems. International Journal of Quantum Information 1(04), 479-506 (2003)

19. Elliott, C.: The darpa quantum network. Quantum Communications and cryptography pp. 83-102 (2006)

20. Furusawa, A., Sørensen, J.L., Braunstein, S.L., Fuchs, C.A., Kimble, H.J., Polzik, E.S.: Unconditional quantum teleportation. Science 282(5389), 706-709 (1998)

21. Gibney, E., et al.: One giant step for quantum internet (2016)

22. Hillery, M., Ziman, M., Bužek, V., Bieliková, M.: Towards quantum-based privacy and voting. Physics Letters A 349(1-4), 75-81 (2006)

23. Horodecki, R., Horodecki, P., Horodecki, M., Horodecki, K.: Quantum entanglement. Reviews of modern physics 81(2), 865 (2009)

24. IBM: Quantum cloud. https://www.ibm.com/cloud/why-ibm/

25. Jennewein, T., Weihs, G., Pan, J.W., Zeilinger, A.: Experimental nonlocality proof of quantum teleportation and entanglement swapping. Physical review letters 88(1), 017903 (2001)

26. Julsgaard, B., Sherson, J., Cirac, J.I., Fiurášek, J., Polzik, E.S.: Experimental demonstration of quantum memory for light. Nature 432(7016), 482-486 (2004)

27. Kimble, H.J.: The quantum internet. Nature 453(7198), 1023-1030 (2008)

28. Kirby, B.T., Santra, S., Malinovsky, V.S., Brodsky, M.: Entanglement swapping of two arbitrarily degraded entangled states. Physical Review A 94(1), 012336 (2016)

29. Ma, X.S., Herbst, T., Scheidl, T., Wang, D., Kropatschek, S., Naylor, W., Wittmann, B., Mech, A., Kofler, J., Anisimova, E., et al.: Quantum teleportation over 143 kilometres using active feed-forward. Nature 489(7415), 269-273 (2012)

30. Megidish, E., Halevy, A., Shacham, T., Dvir, T., Dovrat, L., Eisenberg, H.S.: Entanglement between photons that never co-existed. In: Frontiers in Optics. pp. FTh2C-4. Optical Society of America (2012)

31. Nielsen, M.A., Chuang, I.L.: Quantum computation and quantum information. Quantum 546, 1231 (2010) 
32. Pan, J.W., Bouwmeester, D., Weinfurter, H., Zeilinger, A.: Experimental entanglement swapping: entangling photons that never interacted. Physical Review Letters 80(18), 3891 (1998)

33. Peev, M., Pacher, C., Alléaume, R., Barreiro, C., Bouda, J., Boxleitner, W., Debuisschert, T., Diamanti, E., Dianati, M., Dynes, J., et al.: The secoqc quantum key distribution network in vienna. New Journal of Physics 11(7), 075001 (2009)

34. Pirandola, S., Braunstein, S.L.: Unite to build a quantum internet. Nature pp. 169-171 (2016)

35. Pugh, C.J., Kaiser, S., Bourgoin, J.P., Jin, J., Sultana, N., Agne, S., Anisimova, E., Makarov, V., Choi, E., Higgins, B., et al.: Airborne demonstration of a quantum key distribution receiver payload. In: CLEO: Applications and Technology. pp. ATu4B-5. Optical Society of America (2017)

36. Raussendorf, R., Briegel, H.J.: A one-way quantum computer. Physical Review Letters 86(22), 5188 (2001)

37. Ren, J.G., Xu, P., Yong, H.L., Zhang, L., Liao, S.K., Yin, J., Liu, W.Y., Cai, W.Q., Yang, M., Li, L., et al.: Ground-to-satellite quantum teleportation. arXiv preprint arXiv:1707.00934 (2017)

38. Sasaki, M., Fujiwara, M., Ishizuka, H., Klaus, W., Wakui, K., Takeoka, M., Miki, S., Yamashita, T., Wang, Z., Tanaka, A., et al.: Field test of quantum key distribution in the tokyo qkd network. Optics Express 19(11), 10387-10409 (2011)

39. Sherson, J.F., Krauter, H., Olsson, R.K., Julsgaard, B., Hammerer, K., Cirac, I., Polzik, E.S.: Quantum teleportation between light and matter. Nature 443(7111), 557-560 (2006)

40. Simon, C., Afzelius, M., Appel, J., de La Giroday, A.B., Dewhurst, S., Gisin, N., Hu, C., Jelezko, F., Kröll, S., Müller, J., et al.: Quantum memories. The European Physical Journal D 58(1), 1-22 (2010)

41. Singh, S.K., Srikanth, R.: Generalized quantum secret sharing. Physical Review A 71(1), 012328 (2005)

42. Tindol, R.: Caltech physicists achieve first bona fide quantum teleportation - caltech, http://www.caltech.edu/news/ caltech-physicists-achieve-first-bona-fide-quantum-teleportation-291

43. Vaccaro, J.A., Spring, J., Chefles, A.: Quantum protocols for anonymous voting and surveying. Physical Review A 75(1), 012333 (2007)

44. Van Meter, R.: Quantum networking. John Wiley \& Sons (2014)

45. Yin, J., Cao, Y., Li, Y.H., Liao, S.K., Zhang, L., Ren, J.G., Cai, W.Q., Liu, W.Y., Li, B., Dai, H., et al.: Satellite-based entanglement distribution over 1200 kilometers. Science 356(6343), 1140-1144 (2017)

46. Yin, J., Ren, J.G., Lu, H., Cao, Y., Yong, H.L., Wu, Y.P., Liu, C., Liao, S.K., Zhou, F., Jiang, Y., et al.: Quantum teleportation and entanglement distribution over 100-kilometre free-space channels. Nature 488(7410), 185-188 (2012)

47. Zukowski, M., Zeilinger, A., Horne, M.A., Ekert, A.K.: " event-ready-detectors" bell experiment via entanglement swapping. Physical Review Letters 71(26), 42874290 (1993) 